\title{
Growth of Helicobacter pylori in candle jars
}

\author{
X. G. FAN and T. G. LI \\ Department of Infectious Diseases, Xiangya Hospital, Hunan Medical University, Changsha, Hunan, PR China
}

\begin{abstract}
Culture of Helicobacter pylori requires micro-aerobic conditions which are usually generated by a commercial gas-generator system. However, the commercial gasgenerator pack is not available in most hospitals in developing countries. The present study showed that $H$. pylori grew well in a candle jar system that provided microaerobic condition; this gave similar cultural results to a commercial gas-generator system. Therefore, the candle jar system can be used for $\boldsymbol{H}$. pylori micro-aerobic culture in developing countries where commercial systems are not available.
\end{abstract}

\section{Introduction}

Helicobacter pylori has been implicated in the pathogenesis of gastric carcinoma as well as active chronic gastritis and peptic ulcer disease [1-3]. Since this bacterium was first isolated in 1982, various methods of isolation and subculture for diagnostic and research applications have been documented [4]. The special atmospheric requirements for growth make the culture of $H$. pylori difficult in developing countries. In China, H. pylori infection is diagnosed mostly by serological technique, CLO test or histological stain rather than culture, because of the lack of commercial gas-generator packs to provide micro-aerobic conditions. The urea breath test, which is used widely in developed countries [5], is also not available in the majority of hospitals in developing countries. Because it is difficult to culture $H$. pylori in developing countries, the selection of drug regimens for the treatment of these infections is based on data from developed countries; this may not be appropriate. It has been reported that Campylobacter jejuni and Neisseria gonorrhoeae can grow in candle jars [6], which provide micro-aerobic conditions, as well as in commercial gas incubators. The aim of this small study was to determine whether $H$. pylori could be grown in a sealed candle jar with high humidity.

\section{Materials and methods}

Antral biopsies were obtained from 10 patients with dyspepsia during upper gastrointestinal endoscopy for $H$. pylori histological examination, CLO test and

Received 5 June 1996; revised version accepted 5 Sept. 1996.

Corresponding author: Dr X. G. Fan. culture. Table 1 summarises the characteristics of the cases studied. For the evaluation of the candle jar incubation versus commercial gas-generator incubation systems, equal volumes (c. $\left.1 \times 2 \times 2 \mathrm{~mm}^{3}\right)$ of biopsy specimens were inoculated on to two chocolate agar plates (Columbia agar base, Lab $\mathrm{M}$ ) containing sheep blood $7 \%$ and one plate was incubated in each system. In the commercial gas-generator system (Yiwu company, Zhejiang, China), the plates were incubated at $37^{\circ} \mathrm{C}$ under micro-aerobic conditions in $\mathrm{O}_{2} 5 \%, \mathrm{CO}_{2}$ $10 \%$ and $\mathrm{N}_{2} \quad 85 \%$ with high humidity. A glass anaerobic jar was used in the candle jar system. To ensure high humidity, tissue papers soaked in sterile water were placed in an open plastic petri dish. Inoculated plates, the open petri dish containing wet tissue papers and a lit candle were all placed in the glass jar. The jar was immediately covered with its lid, completely sealed with vaseline and incubated at $37^{\circ} \mathrm{C}$ for up to 7 days. After incubation for 3 days the plates were examined for growth daily. If no growth of H. pylori was observed after incubation for 7 days, the plates were discarded.

Each of six strains of $H$. pylori, stored in Brain-heart Infusion Broth (Unipath) with sheep blood 7\% at $-70^{\circ} \mathrm{C}$, was subcultured separately in both systems under the same conditions as described above.

Gram's stain, urease and catalase tests were used to confirm the identification of $H$. pylori isolates and to exclude the possibility of contamination.

A disk-diffusion test was performed to validate the candle jar system for drug susceptibility testing. One strain of $H$. pylori, which had been confirmed previously to be susceptible to metronidazole, was subcultured on to six chocolate agar plates and a 6-mmdiameter disk containing metronidazole (Hangzhou 
Table 1. Characteristics of 10 patients investigated for $\mathrm{H}$. pylori

\begin{tabular}{llllll}
\hline $\begin{array}{l}\text { Patient } \\
\text { no. }\end{array}$ & Sex & $\begin{array}{c}\text { Age } \\
\text { (years) }\end{array}$ & OGD & $\begin{array}{c}\text { CLO } \\
\text { test }\end{array}$ & Histology \\
\hline 1 & $\mathrm{M}$ & 54 & GST + DU & + & Acute and chronic inflammation, HP seen \\
2 & $\mathrm{M}$ & 41 & GST + DU & + & Acute and chronic inflammation, HP seen \\
3 & $\mathrm{~F}$ & 40 & GST & + & Acute and chronic inflammation, HP seen \\
4 & $\mathrm{M}$ & 28 & GST + DU & + & Chronic inflammation, HP seen \\
5 & $\mathrm{M}$ & 38 & Normal & - & Normal \\
6 & $\mathrm{~F}$ & GST & + & Chronic inflammation, HP seen \\
7 & $\mathrm{M}$ & GS & GST & + & Chronic inflammation, HP seen \\
8 & $\mathrm{M}$ & 50 & GST + DU & + & Chronic inflammation, HP seen \\
9 & F & 32 & GST & + & Chronic inflammation, HP seen
\end{tabular}

OGD, oesophageal, gastric and duodenal status; GST, gastritis; DU, duodenal ulcer; HP seen, $H$. pylori was identified histologically by haematoxylin and eosin stain.

Chemicals Company, Zhejiang, China) $5 \mu \mathrm{g}$ was applied to each plate; three plates were incubated in each culture system. Diameters of zones of growth inhibition were measured at 3,4 and 5 days.

\section{Results and discussion}

Similar culture results were obtained in both systems. Of 10 biopsy samples, eight showed profuse growth of $H$. pylori colonies in the commercial gas-generator incubation system and seven grew well after incubation in the candle jar system. Compared with the results of $H$. pylori histological examination (Table 1), the sensitivity of $H$. pylori culture in the commercial gas-generator system in the present study was $89 \%(8$ of 9). This result is agreement with those previously described [4]. All six stored H. pylori strains grew well in both systems. However, after incubation for 3 days the colonies from both biopsy specimens and stored strains observed in the candle jar were smaller than those observed in the commercial system. Colonies of the same size as those observed after incubation with a gas-generator $(0.5-1 \mathrm{~mm}$ diameter) were obtained after culture for 5 days in a candle jar.

There was no difference between the sizes of the zone of growth inhibition obtained with metronidazole in the two systems after incubation for 3, 4 and 5 days.

Although $H$. pylori infection may be diagnosed by serological detection, breath test, rapid urease exam- ination or histological stain, culture is still required to determine antimicrobial susceptibility and to obtain the organism for typing purposes. However, microaerobic culture of micro-organisms with commercial gas-generator systems is not available in some developing countries, making the selection of appropriate antimicrobial agents for the treatment of infections with $H$. pylori difficult. In the candle jar system described in the present study, H. pylori grew successfully and disk diffusion test results equivalent to those from the commercial system were obtained. We believe that this system will help to isolate $H$. pylori from patients with gastritis or peptic ulcer, and should enable drug susceptibility testing where commercial gas-generator systems are not available.

\section{References}

1. Fan X-G, Chua A, Fan X-J, Keeling PWN. Lipid peroxidation induced by Helicobacter pylori. Exp Clin Gastroenterol 1994; 4: 29-34.

2. Fan X-G, Chua A, Fan X-J, Keeling PWN. Increased gastric production of interleukin- 8 and tumour necrosis factor in patients with Helicobacter pylori infection. $J$ Clin Pathol 1995; 48: $133-136$.

3. Fan X-G, Kelleher D, Fan X-J, Xia HX, Keeling PWN. Helicobacter pylori increases proliferation of gastric epithelial cells. Gut 1996; 38: 19-22.

4. Marshall BJ. Helicobacter pylori. Am J Gastroenterol 1994; 89 Suppl 8: S116-S128.

5. Rune SJ. Diagnosis of Helicobacter pylori infection. When to use which test and why. Scand J Gastroenterol 1996; 31 Suppl 215: $63-65$.

6. Birkenmeyer L, Armstrong AS. Preliminary evaluation of the ligase chain reaction for specific detection of Neisseria gonorrhoeae. J Clin Microbiol 1992; 30: 3089-3094. 